\title{
Critical Upper Limb Ischemia due to Subclavian Artery Compression Caused by Initially Undiagnosed Cervical Rib - A Case Report

\author{
SULTAN MAHMUD ${ }^{1}$, OMAR SADEQUE KHAN ${ }^{1}$, ABDULLAH AL MAMUN ${ }^{2}$, FIDAH HOSSAIN², \\ MD AFTABUDDIN ${ }^{1}$, ASIT BARAN ADHIKARY ${ }^{1}$, SMG SAKLAYEN $^{1}$ \\ ${ }^{1}$ Department of cardiac surgery , Bangabandhu Sheikh Mujib Medical University( BSMMU), Dhaka, ${ }^{2}$ Department of vascular \\ surgery, National Institute of Cardiovascular Disease (NICVD), Dhaka.
}

Address of Correspondence: Professor Dr. Asit Baran Adhikary, Chairman, Department of Cardiac Surgery, Bangabandhu Sheikh Mujib Medical University, Dhaka. E-mail: drasit2005@yahoo.com

\begin{abstract}
True subclavian artery aneurysms are relatively rare events. Thoracic outlet compression is responsible for $75 \%$ of those aneurysms. They are formed as a result of compression of subclavian artery, for example a cervical rib.

A case of subclavian artery aneurysm secondary to cervical rib in a 35 year old young adult, who presented with a critical ischemia in his dominant right upper limb. Plain x-ray of cervical spine revealed bilateral cervical ribs and duplex study of the both upper limb arteries concluded aneurysmal dilatation of mid-distal subclavian artery of both sides with mural thrombus on the right side, marked distal ischemia in the right upper limb due to occlusive thrombus in the distal arterial tree, normal distal arterial flow in the left upper limb.
\end{abstract}

Although it is a rare lesion, cervical rib leading to thoracic outlet compression should always be included in the differential diagnosis of a critically ischemic limb in young age group. Surgical management should be considered in a patient with subclavian artery aneurysm due to cervical rib to prevent additional embolic events.

Key words: Thoracic outlet syndrome (TOS), cervical rib, subclavian artery aneurysm, critical ischemia of upper limb.

Introduction:

Thoracic outlet syndrome (TOS) was introduced by Peet in 1956 to put together upper limb symptoms arising from neurovascular compression in the interscalene triangle ${ }^{1}$. It is classified into three categories: arterial (subclavian artery, 1\%), venous (subclavian vein, 4\%-6\%) and neurological (brachial plexus, 94\%-97\%). Arterial TOS is rare and typically caused by subclavian artery compression in association with a cervical rib or first rib anomaly ${ }^{2}$. Due to the compression of subclavian artery by cervical rib there is turbulent flow of blood through the narrowed segment and this type of long standing stress eventually results in degeneration of arterial wall and formation of aneurysm and thrombosis. This results in restricted blood flow to the limb. In the long run a critically ischemic upper limb developed. Prompt recognition of this type of compression by cervical rib is extremely important, as delays can lead to severe ischemic complications like, tissue loss requiring debridement or amputation and permanent disability. The objective of this paper is to provide a scenario of a young male with critically ischemic right upper limb caused by undiagnosed compression of subclavian artery by an accessory cervical rib.

\section{Case report:}

A thirty five years old normotensive, nondiabetic, male smoker presented with a six months history of pain in the right upper limb with paresthesia and blackening of the tip of the right index and ring fingers (figure-1). The pain was intermittent, dull aching in nature, increased on working and relieved by rest. Initially the pain used to relieve with

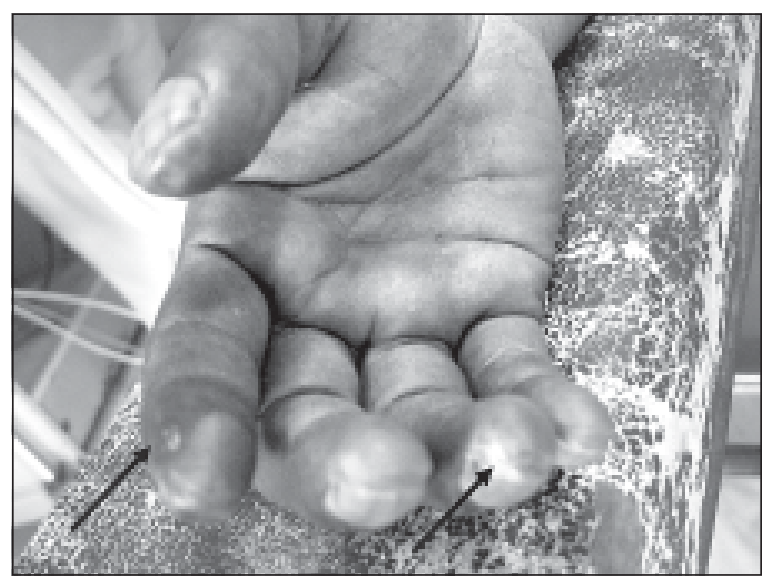

Fig.-1: Blackening of the tips of right index and ring fingers. 
analgesics but gradually has become intractable. This was associated with coldness, numbness and blackening of the finger tips particularly right index and ring fingers. He worked in a bakery as a delivery man (manual worker) and he had no other history relevant to this condition. Clinical examination revealed right upper limb pulses were not palpable, pulsatile swelling in the right supraclavicular region and dry gangrene involving the tip of the right index and ring fingers. Other peripheral pulses were palpable and normal.

Investigation profile, plain $\mathrm{X}$-ray cervical rib A/P view revealed bilateral cervical rib (figure-2) and early degenerative changes of cervical spines. Color duplex ultrasound scanning of the both upper limb arteries showed that aneurysmal dilatation of the distal part or third part of subclavian artery (diameter about 13x12mm) with mural thrombus on right side and normal on left side.

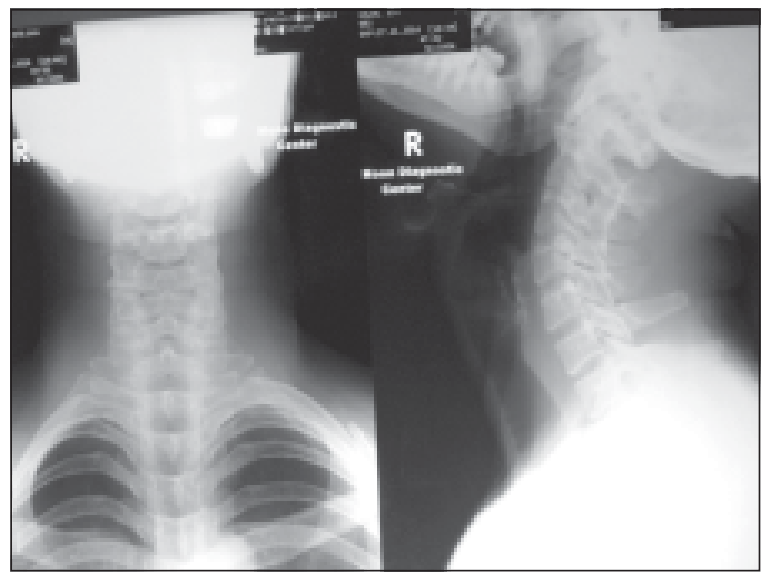

Fig.-2: Plain X-Ray Cervical rib A/P View.

The patient was treated surgically. With all aseptic precaution under general anaesthesia, proper positioning and draping was done. Supraclavicular transverse incision was made and sclenus anterior muscle (right) was incised. Right subclavian artery was identified and control taken both proximally and distally. Heparinization done then thrombectomy, Fogerty embolectomy and aneurysmorraphy of right subclavian artery was done (figure-3). Fogerty embolectomy of right brachial artery was also done. (Figure-4).

The patient was discharged on eighth post operative day with slight cyanosis on right hand. Tip of index and ring finger was minimally gangrenous, motor and sensory function of right hand was improving. He was then referred to orthopaedic consultant for the management of gangrenous finger. During discharge double antiplatelet treatment was prescribed: Acetylsalicylic acid at a dose of

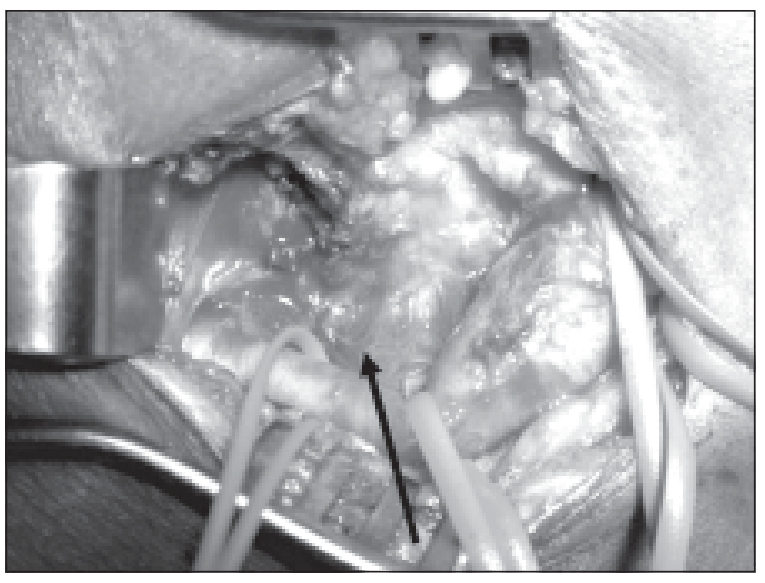

Fig.-3: Aneurysm of right subclavian artery.

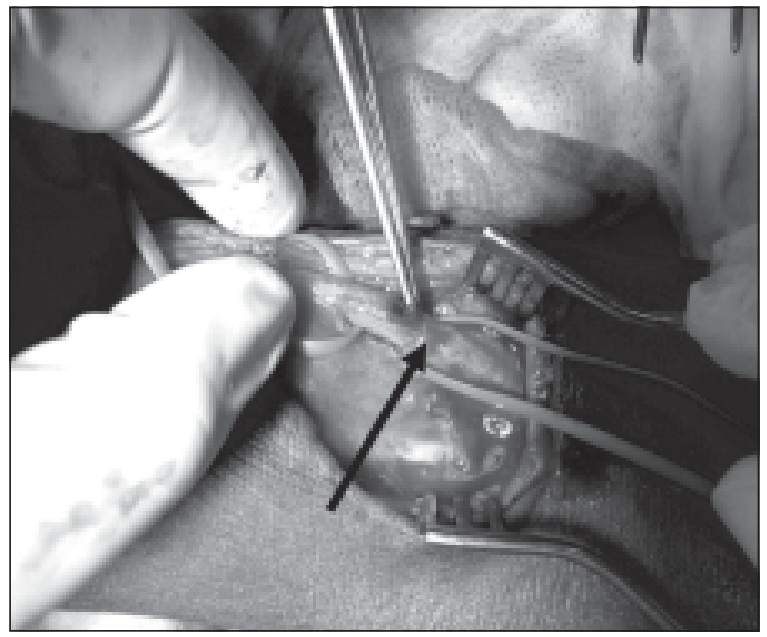

Fig.-4: Thrombosed Brachial Artery

$75 \mathrm{mg}$ daily and clopidogrel at a dose of 75mg daily for two months. He was advised for follow up after one month.

\section{Discussion:}

In this case, the patient presented with six months intermittent right upper extremity claudication due to pressure of cervical rib upon right subclavian artery leading to aneurysm and thrombosis. This is unusual in 35 year old man. True subclavian artery aneurysms are relatively rare lesion, accounting for approximately $0.13 \%$ of all aneurysms ${ }^{3,4}$. Thoracic outlet compression is responsible for $75 \%$ aneurysm which arise from the distal part of subclavian artery, also known as extra thoracic subclavian artery or subclavian axillary artery aneurysm ${ }^{5,6}$.

Thoracic outlet syndrome (TOS) or thoracic outlet compression syndrome is due to compression of cervical vessels or brachial plexus in the region of thoracic outlet. Congenital causes include cervical rib, anomalous first rib 
or fibromuscular band. Acquired causes are blunt trauma, clavicle deformities, scalene musculotendinous hypertrophy and subclavian artery aneurysms ${ }^{7}$. Less than $5 \%$ patients with upper extremity ischemia is due to TOS, of this cervical rib is the most common, especially in fit young people ${ }^{8}$.About $70 \%$ cases of TOS are associated with neurological symptoms and when vascular injury occurs, venous problems for example axillary vein thrombosis are more common than arterial. TOS is becoming more common in athletes, swimmers, who perform relative over hand movement and those who perform hyper abduction movement ${ }^{9}$. Our patient is a manual worker. He used to carry heavy boxes over his head, supported by his hands.

Cervical rib represents an anomalous development of a rib, usually from the seventh cervical vertebra ${ }^{10}$ and may be associated with spinal anomalies elsewhere. The embryonic formation of cervical ribs is attributed to a conflict between forming ribs and plexus ${ }^{11}$.It has been reported that a cervical rib present in less than $1 \%$ of the population, they are asymptomatic in $90 \%$ cases $^{12,13}$. Durham et al. (1995) found that sixteen (73\%) of twenty two patients with subclavian artery compreesion had cervical ribs and in five (3\%) of those were bilateral ribs ${ }^{14}$. Cervical ribs are two types : complete or incomplete. Only complete cervical ribs have been reported to produce vascular symtoms ${ }^{15}$. In the present case most likely predisposing factor of subclavian artery compression and aneurysm was the presence of anomaly.

Pain in the arm and hand is the presenting symptoms in subclavian artery compression which typically presents in young's, otherwise healthy people with vigorous shoulder activity. Common findings on physical examination consist of pulse less, pale and cold distal upper limb. One must have high suspicion for presence of cervical ribs when dealing with patients complaining of unilateral upper limb sensory and motor symptoms ${ }^{16}$. Unfortunately this patient had not received a proper initial physical assessment with a follow up plan and returned with a critically ischemic limb for surgical interventions. In order not to delay the diagnosis and correct treatment of arterial compression the clinician must differentiate such arm ischemia from Reynaud's syndrome, vaso-spasmotic diseases, distal small artery occlusive diseases or proximally large artery occlusion which may result in this symptom complex.

The lumen of subclavian artery becomes constricted owing to compression in the base of intersclaene triangle in which subclavian artery pass. Chronic arterial spasm induced by the pressure of cervical rib may increase flow velocity and shear stress at the affected arterial wall. These mechanical factors may damage the intima and trigger clotting factors followed by aneurysm and thrombus formation. Further arterial compression with increase in symptoms can occur during rotation and elevation of $\operatorname{arm}^{16}$.

Plain X-ray of neck and duplex study of both upper limb arteries were used to identify bony abnormalities and confirm the presence of an aneurysm and thrombosis. However CT and MRI imaging angiography remain the investigation of choice ${ }^{5,6}$. In this patient plain X-ray cervical rib A/P view revealed bilateral cervical rib (figure2) and early degenerative changes of cervical spines. Color duplex ultrasound scanning of the both upper limb arteries showed that aneurysmal dilatation of the distal part or third part of subclavian artery (diameter about 13x12mm) with mural thrombus on right side and normal on left side.

Cervical rib with subclavian artery thrombosis and aneurysm is difficult to treat. Surgical repair is the standard management, even for asymptomatic subclavian artery aneurysm due to increase in size and rupture. These involve resection of aneurysm and reconstruction of the artery with or without graft insertion. Decompression of TOS with resection of cervical rib and first rib, anterior scalene muscle or constricting fibrous band or median sternotomy, lateral thoracotomy may be necessary for intrathoracic aneurysm ${ }^{17}$. It must be emphesised that it is a technically demanding procedure that requires very good knowledge about anatomy of this region and precise surgical technique, since there is a high risk of injury in such structure and subclavian artery, vein and brachial plexus, phrenic nerve and thoracic duct ${ }^{18}$. Distal arterial occlusion secondary to embolic will warrant angioplasty or thromboembolectomy to restore patency of vessels. Successful management of this patient involves multidisciplinary approach.

\section{Conclusion:}

Cervical rib leading to thoracic outlet compression should be included in the diagnosis of a critically ischemic limb in young age group. Vascular complication associated with cervical rib can be limb threatening. This report demonstrates the importance and difficulty of correctly diagnosing a rare pathology that has a common presentation. Surgical management should be considered in a patient with subclavian artery aneurysm and thrombosis due to presence of cervical rib to prevent additional embolic complications. 


\section{References:}

1. sPeet RM, Hendouckesen JD, Anderson TP, Martin GM: Thoracic outlet syndrome: evaluation of a therapeutic exercise program. Proc staff Meet Mayo clinic 1956; 31: 281-87.

2. Story of arterial TOS patient treated by Robert Thompson, MD, at the Washington University Center for Thoracic Outlet Syndrome at Barnes-Jewish Hospital.

3. Pagni S, DenataleRW, Femeini AM: Atherosclerotic aneurysm of the proximal subclavin artery: a case report. Ann surgery 1998; 64: 323-27.

4. Doglus DA, Adelman MA, Esposito R, Rockman C: Surgical repair of a left subclavian artery aneurysm causing stenosis of a left internal mammary graft: a case report. Vascular and endovascular surgery 2005; 39: 281-85.

5. Rutherford RB: vascular surgery, $6^{\text {th }}$ edition America: Elsevier Saunders, 2005: 1552-57.

6. Hallett JW, Mills JL, Earnshaw J, Reekers JA: comprehensive vascular and endovascular surgery 2004; 485-89.

7. Casey RG, Richards S, Donohoe MO: Exercise induced critical ischemia of the upper limb secondary to a cervical rib. $\mathrm{Br} \mathrm{J}$ Sports Med 2003; 37: 455-56.

8. Aburahma AF, White JF: Thoracic outlet syndrome with arm ischemia as a complication of cervical rib. W V Medical Journal 1995; 91: 92-94.

9. Richardson AB: thoracic outlet syndrome in aquatic athletes. Clinical sports med 1999; 18: 361-78.
10. Tubbs RS, Muhleman M, Miller J, Shoja MM, Loukas M, Welson JC et al Cervical ribs with neurological sequelae in children: A case series Childs Neuv Syst 2011.

11. Jones FW: The Anatomy of cervical ribs- Proc R Soc Med 1913; 6: 95-113.

12. Sanders RJ, Hammond SL: Management of cervical ribs and anomalous first ribs causing neurogenic thoracic outlet syndrome. Vasc Surg 2002; 36: 51-56.

13. Davidovic LB, Kustic DM, Jakovijevic NS,Kuzmanovic JL, Simic TM: Vascular thoracic outlet syndrome. Would J Surgery 2003; 27: 545-50.

14. Durham JR, Yao JS, Pearce WH, Nuber GM, McCarthy WJ: Arterial injuries in the thoracic outlet syndrome. Vascular surgery 1995; 21: 57-69.

15. Short DW: The subclavian artery in 16 patients with complete cervical ribs. Journal cardiovascular surgery1975; 16: 135-41.

16. Jusufovic M, Sanset EC, popperud TH, Solberg S, Ringstad G and Kerty E: Anusual case of the syndrome cervical artery thrombosis and cerebeller and cerebral infarction. BMC Neurology2012; 12: 48-49.

17. Leemput AV, Maleux G, Heye S, Nevelsteen A: Combined open and endovascular repair of true right subclavian artery aneurysm without proximal neck. Interact cardiovascular \& Thoracic surgery 2007; 6: 406-08.

18. Dzieciuchowicz L, Wlodarczyk W, Oszkinis G:Critical upper limb ischemia caused by initially undiagnosed thoracic outlet syndrome-case report. Polski Przegland Chirurgiczny 2012; 84: $158-62$. 